\title{
Norois
}

Environnement, aménagement, société

$217 \mid 2010 / 4$

Interroger les processus de valorisation des espaces urbains

\section{La récupération du centre ancien de Barcelone depuis le début des années 1980. Enjeux, processus, contradictions}

The recuperation of the old city-centre of Barcelona since the early eigthies. Issues, processes, contradictions

\section{Hovig Ter Minassian}

\section{(2) OpenEdition}

\section{Journals}

\section{Édition électronique}

URL : https://journals.openedition.org/norois/3437

DOI : $10.4000 /$ norois.3437

ISBN : 978-2-7535-1565-9

ISSN : $1760-8546$

\section{Éditeur}

Presses universitaires de Rennes

Édition imprimée

Date de publication : 15 décembre 2010

Pagination : 13-27

ISBN : 978-2-7535-1340-2

ISSN : 0029-182X

\section{Référence électronique}

Hovig Ter Minassian, «La récupération du centre ancien de Barcelone depuis le début des années 1980. Enjeux, processus, contradictions », Norois [En ligne], 217 | 2010/4, mis en ligne le 31 décembre 2012, consulté le 14 janvier 2022. URL : http://journals.openedition.org/norois/3437 ; DOI : https:// doi.org/10.4000/norois.3437 


\title{
LA RÉCUPÉRATION DU CENTRE ANCIEN DE BARCELONE \\ DEPUIS LE DÉBUT DES ANNÉES I980 \\ Enjeux, Processus, Contradictions
}

\author{
Hovig Ter Minassian \\ CITERES UMR 6173 \\ (Université François-Rabelais de Tours), \\ 33, allée Ferdinand-de-Lesseps, BP 60449 - 37204 Touns cedex 03, France \\ hovig.terminassian@univ-tours.fr
}

\section{RÉSUMÉ}

Le centre ancien de Barcelone (qui correspond au district de "Ciutat Vella ») a fait l'objet d'une politique publique de réhabilitation particulièrement énergique depuis le début des années 1980. Elle a entraîné ou soutenu la diffusion de processus de valorisation et de dévalorisation multiples et parfois contradictoires avec les attentes des résidents. Le centre ancien de Barcelone apparaît ainsi comme fragmenté par de multiples processus : gentrification, désembourgeoisement, marginalisation. À travers une analyse de ces différents processus et de deux projets publics d'aménagement qui ont été menés en parallèle (Rambla del Raval et Pou de la Figuera), il est possible d'étudier le rôle des politiques publiques dans la "récupération » du district de Ciutat Vella à Barcelone et de mieux comprendre les incidences des choix d'aménagement et d'urbanisme pris par la Municipalité de Barcelone sur l'évolution récente du centre ancien de l'agglomération catalane.

MotS CLÉ : Barcelone - Ciutat Vella - Raval-gentrification - désembourgeoisement - politique de réhabilitation - urbanisme

\footnotetext{
ABSTRACT

The recuperation of the old city-centre of Barcelona since the early eigthies. Issues, processes, contradictions

The old city-centre of Barcelona (which corresponds to the district of "Ciutat Vella") has been the subject, since the beginning of the 1980s, of a public rehabilitation policy which was particularly vigorous. It has led or supported the emergence of multiple processes of valorisation and devaluation, and sometimes contradictory with the expectations of the residents. Thus, the old city-centre of Barcelona appears to be fragmented by multiple processes (gentrification, "desembourgeoisement", marginalization). Through an analysis of these various processes and of two public urban planning projects carried out in parallel (Rambla del Raval and Pou de la Figuera), it is possible to study the role of local public policies in the "recovery" of the Barcelona's district of Ciutat Vella, and a better understanding of the implications of the Municipality of Barcelona's urban planning and urbanism policies on the recent evolution of the old centre of the Catalan agglomeration.
}

KEY WORDS : Barcelona-Ciutat Vella-Raval-gentrification-désembourgeoisement - rehabilitation policy - urbanism 
À partir du début des années 1980, face à la « crise urbaine » (Lévy, 2003) qui le touchait et dans un contexte de retour à la démocratie, le centre ancien de Barcelone (qui correspond au district de « Ciutat Vella », ou « Vieille Ville » en catalan) a fait l'objet d'une politique publique de réhabilitation particulièrement énergique. Nous parlerons de « récupération » pour désigner cette revitalisation urbanistique, démographique, économique, et symbolique des quartiers anciens de la capitale catalane. Comme le rappelle Alain Bourdin, les «mots » de la réhabilitation sont très nombreux mais rarement neutres (Bourdin, 1984). Le terme de « récupération » désigne d'abord le réinvestissement du quartier par les politiques de réhabilitation urbaine, mais sans préjuger des moyens mis en œuvre, de la rénovation ou démolition/reconstruction à la réhabilitation, avec toute la gamme des possibles entre les deux. Ensuite, il renvoie au réinvestissement socio-économique et culturel des quartiers anciens par les classes moyennes ou aisées, sans préjuger non plus des modalités de ce retour (gentrification, spéculation immobilière). Enfin, le terme de « récupération » vise à prendre en compte la dimension symbolique de la requalification des espaces centraux. La revitalisation du centre ancien de Barcelone s’inscrit en effet dans une volonté, au début des années 1980, aussi bien de la part des mouvements sociaux urbains que de la nouvelle équipe municipale, de « récupérer » l'initiative de l'action urbanistique et de réinvestir des secteurs malmenés par les politiques d’urbanisme de la période franquiste.

Ce réinvestissement est parfois en contradiction avec les attentes, d'une part des résidents du centre ancien, dont le maintien est menacé par la hausse des prix immobiliers et par la disparition du petit commerce au profit des magasins de mode et de restaurants touristiques, et d'autre part des immigrants qui ont longtemps privilégié les logements bon marché des quartiers dégradés du cœur de l'agglomération catalane. L'analyse que nous proposons de mener doit permettre d'examiner de manière critique les incidences des politiques municipales d'urbanisme dans la diffusion des différentes formes de réappropriation des quartiers hérités et dans les contradictions qu'elles révèlent, à travers l'exemple du district de Ciutat Vella de Barcelone. Nous entendons par réappropriation le fait que les quartiers anciens sont désormais investis par des populations nouvelles, qu'ils soient des gentrifieurs ou des immigrants étrangers pauvres, et dont les pratiques se superposent aux anciennes, entrant parfois en opposition avec ces dernières. Les pouvoirs publics sont des acteurs à part entière de ces réappropriations multiples, et les choix qu'ils opèrent en matière de projet d'aménagement ou de grandes orientations urbanistiques ont des conséquences sur les processus en présence. Ce type d'analyse a déjà été mené dans les études sur la rénovation urbaine ou sur la gentrification dans les villes anglo-saxonnes et européennes (Coing, 1966; Castells, 1974 ; Bailey et Robertson, 1997 ; Clerval, 2008), mais le cas de Barcelone reste peu étudié alors même que les enjeux de la réhabilitation de son centre ancien offre des perspectives d'étude originales.

Dans un premier temps, nous présenterons la multiplicité des enjeux de la récupération de Ciutat Vella. Ils déterminent la politique de réhabilitation adoptée par la Municipalité à partir des années 1980. Puis, nous étudierons les principales trajectoires socio-démographiques qui ont caractérisé l'évolution du district de Ciutat Vella depuis que cette politique est menée. Enfin, nous tenterons de comprendre le rôle des pouvoirs publics dans les différentes modalités de ce réinvestissement, et dans les tensions qu'elles génèrent, à travers l'analyse des principales opérations d'aménagement dans les secteurs de la Rambla del Raval et de Pou de la Figuera. À Ciutat Vella, les politiques publiques d’urbanisme participent d'une stratégie de normalisation socio-spatiale du tissu urbain de quartiers anciens.

\section{Une politique énergique à l'égard des quartiers anciens dégradés}

À la fin des années 1980, le centre ancien de Barcelone présente tous les aspects d'un quartier historique et populaire au tissu urbain dégradé, à l'exception des quartiers plus bourgeois situés le long de la Via Laietana. Il continue pourtant à jouer un rôle particulier dans les fonctions récréatives et culturelles à l'échelle de la ville et dans l'imaginaire des Barcelonais ${ }^{1}$.

1. Le district est lui-même découpé en quatre quartiers relativement distincts par leur histoire, leur tissu socio-économique et leur morphologie urbaine : Barceloneta, Parc, Gótic, Raval. 


\section{La CRise urbaine de Ciutat Vella au MOMent du Retour À la démocratie}

Les enjeux de la politique de réhabilitation sont d'abord démographiques. Le district de Ciutat Vella connaît depuis les années 1950 une chute de sa population et son vieillissement, que l'immigration - nationale des années 1960 et 1970, puis internationale à partir des années 1980 et 1990 - a à peine compensés. Le centre ancien comptait encore environ 258000 habitants en 1955, mais seulement 116874 en 1981 (Gomà, 1997), alors que pour la même période la commune de Barcelone connaissait une croissance de $+24,9 \%$. Cette baisse s'explique notamment par la dégradation du tissu urbain, la crise des activités traditionnelles du centre ancien et les opérations de rénovation qui ont chassé une partie des habitants. Au milieu des années 1980, la part des personnes âgées (65 ans ou plus) s'élevait à environ 23,6\% de la population de Ciutat Vella contre $15 \%$ seulement pour la commune de Barcelone (Garcia i Miquel et Vilanova i Claret, 1991).

Les enjeux sont également socio-économiques. Au début des années 1980, Ciutat Vella était l'un des principaux quartiers ouvriers de Barcelone. En 1970, le centre ancien de Barcelone comptait $58,2 \%$ de " cols bleus " (artisans, ouvriers et autres travailleurs du secteur secondaire), ce qui le classait parmi les quartiers les plus ouvriers de Barcelone (Ajuntament de Barcelona, 1970), avec des disparités fortes à l'échelle du centre ancien, les classes supérieures étant surreprésentées dans le Barrio Gótico et le Casc Antic par rapport à la moyenne du district. La crise de l'emploi a entraîné un taux de chômage rapporté à la population active particulièrement fort, qui dépassait les $30 \%$ (contre $21,4 \%$ dans la commune de Barcelone) $)^{2}$.

La marginalisation socio-économique des populations du centre ancien se conjuguait avec la marginalisation spatiale d'un tissu urbain dégradé. Au début des années 1980, 12 \% des logements étaient privés d'eau courante et les installations de gaz, d'eau et d'électricité étaient généralement en mauvais état, lorsqu'elles existaient. On estimait que $20 \%$ des logements ne disposaient pas de douche, et que $14 \%$ d'entre eux n'avaient pas de toilettes privées, contre 2,65\% seulement dans l'ensemble de la commune de Barcelone (Gomà, 1997). Les logements de la Barceloneta étaient particulièrement petits, du fait de leurs redécoupages successifs depuis le XVIII ${ }^{\mathrm{e}}$ siècle.

Enfin, les enjeux de la récupération sont politiques. La fin des années 1970 est marquée par un tournant dans l'histoire des villes espagnoles. La fin du régime franquiste et l'adoption d'une nouvelle Constitution en 1979 rendent aux Municipalités la capacité d'aménager l'espace urbain. À Barcelone même, les élections municipales portent au pouvoir local une coalition de gauche aux ressources financières limitées, mais particulièrement ambitieuse dans son projet de ville, notamment celui de la revalorisation de son centre ancien, longtemps considéré comme à la fois populaire et mal famé ${ }^{3}$. Ce contexte très particulier explique la volonté de la Municipalité de rompre avec les politiques urbanistiques de l'époque franquiste pour le centre ancien (manque de construction de logements sociaux, opérations spéculatives de rénovation, stratégies de pourrissement).

\section{L'ÉLABORATION D’UN « MODÈLE » DE RÉHABILITATION DU CENTRE ANCIEN}

Pour répondre à ces nombreux enjeux, la Municipalité a progressivement élaboré un modèle d'intervention urbaine à Barcelone (ou Modelo Barcelona), structuré autour de quelques grands principes : priorité accordée à la requalification des espaces publics, multiplication d'opérations ponctuelles plutôt que quelques grands projets qui auraient accaparé toutes les ressources finan-

2. Ici et dans le reste de notre texte, la population active comprend à la fois les actifs ayant un emploi et ceux au chômage.

3. S. Savary, dans sa thèse sur les paysages de Barcelone (2005), montre que pendant longtemps, la mauvaise réputation du «Barrio Chino » (quartier aux limites relativement floues mais correspondant plus ou moins à une grande moitié sud du Raval) a eu tendance à se diffuser à l'ensemble du district de Ciutat Vella. Le centre ancien fut ainsi durant tout le xx siècle considéré comme un haut lieu de la prostitution et des trafics en tout genre. Dans le même temps, il a gardé l'image d'un quartier à la fois populaire, laborieux et festif, avec ses théâtres et ses cabarets - qui s'oppose dans l'imaginaire barcelonais aux quartiers tranquilles et bourgeois de l'Ensanche - mais aussi celle d'un haut lieu de l'agitation politique et révolutionnaire, qui n’a pas hésité à prendre les armes pour défendre la République lors de la guerre civile. 
cières, ou encore effet «boule de neige » escompté de la part des investissements publics sur les investissements privés. Ce Modelo Barcelona a progressivement permis de structurer le marketing urbain de l'agglomération. Il a été porté par des hommes politiques comme Pasqual Maragall, maire de Barcelone de 1982 à 1997, ou des architectes comme Oriol Bohigas, directeur des services d’urbanisme de la Municipalité jusqu’en 1984 puis conseiller du Maire. Dans les années 1990, le Modelo Barcelona a connu un réel succès sur le plan international, la Municipalité de Barcelone ayant reçu en 1999 la prestigieuse médaille du Royal Institute of British Architects (RIBA), et sa politique étant également devenue une référence pour d'autres villes (notamment en Amérique du Sud).

Les critiques du Modelo Barcelona ont été nombreuses depuis la fin des années 1990, aussi bien de la part des résidents, des riverains, directement concernés par la politique de réhabilitation, que de la part de professionnels, géographes, sociologues, architectes, urbanistes, qui se sont interrogés sur la distorsion entre l'image quasi idyllique que les discours institutionnels cherchent à donner des différents programmes urbanistiques, et la réalité (Von Heeren, 2002; Borja, 2004 ; Capel, 2005; Andreu, 2008). Il existe cependant un certain déficit d'analyse précise des effets du Modelo Barcelona sur le tissu socio-démographique du centre ancien. L'une des réussites affichées par les pouvoirs publics est justement d'avoir su réhabiliter le district de Ciutat Vella sans provoquer sa gentrification.

Forgé par Ruth Glass en 1964 dans son étude sur Londres, le terme décrit la substitution dans certains quartiers des classes moyennes ou supérieures aux classes populaires, s'accompagnant de la réhabilitation du parc de logements (Glass, 1964). Il convient cependant de souligner deux particularités du contexte barcelonais. D’abord, si la gentrification souffre encore d'un faible nombre d'études concernant le contexte espagnol en général et barcelonais en particulier, l'usage qui en est fait peut parfois surprendre dans les publications municipales à Barcelone. Il est très peu fait mention d'éventuels processus de gentrification dans les publications institutionnelles réalisées sous la direction de personnalités universitaires, sous ce terme ou sous un synonyme ne relevant pas forcément du vocabulaire « technique » du chercheur en sciences sociales. Ceci peut dénoter une absence de volonté de tirer les leçons des expériences menées dans d'autres pays, ou au contraire le souci d'éviter un débat polémique, même si, dès 1983, O. Bohigas pose le problème et propose de le résoudre par une forte intervention publique dans le secteur de la construction de logements. Parfois, au contraire, par un glissement subtil de sens, le processus de gentrification est présenté comme un atout majeur pour le devenir de Ciutat Vella sans que soient mises en débat toutes ses implications (Ajuntament de Barcelona, 1991). Dans un ouvrage publié par la Municipalité de Barcelone, qui fait le bilan de ses politiques urbanistiques à Ciutat Vella dans les années 1980 et 1990, la gentrification y est réduite à un simple processus de « reprise » démographique. Une définition apparaît bien à la toute dernière page (et en note infrapaginale), bien qu'elle soit sommaire : "expulsió d'habitants per la irrupció de noves clases benestants » (Procivesa, 2003) ${ }^{4}$. Mais l'ouvrage, beaucoup plus riche en photographies qu'en analyses statistiques précises sur la question, affirme en conclusion que la politique de réhabilitation « ha evitat l'expulsió de residents i ha creat un paradigma de renovació urbana : transformació sense trencament ni gentrificació » (Procivesa, 2003) $)^{5}$. Qu'en est-il réellement?

La seconde particularité du contexte barcelonais est que si les analyses à l'échelle de l'ensemble du district de Ciutat Vella sont très nombreuses, et mettent en avant les progrès considérables réalisés depuis le début des années 1980, tant en termes de logements réhabilités, de hausse du niveau moyen d'instruction ou d'élévation du niveau de vie, les analyses et les représentations cartographiques sont beaucoup plus rares à une échelle micro-urbaine. Cette constatation nous a conduit à privilégier une échelle d'analyse plus fine en faisant l’hypothèse que la réalité était sans

4. «Expulsion des habitants du fait de l'arrivée de nouvelles classes à plus forte capacité économique ».

5. « a évité l'expulsion des résidents et a créé un paradigme de la rénovation urbaine : une transformation sans fracture ni gentrification ". 
doute contrastée, révélant à la fois des processus d'amélioration des conditions de vie, mais aussi, dans d'autres secteurs, le maintien de zones dégradées. C'est pourquoi, il convient d'analyser dans un second temps la multiplicité des transformations socio-démographiques du centre ancien de Barcelone depuis l'initiation de sa politique de réhabilitation.

\section{Ciutat Vella entre gentrification et marginalisation 1991-2005}

En nous appuyant sur une série d'analyses statistiques univariées et plurivariées, nous avons cherché à mettre en évidence la multiplicité des processus à l'œuvre dans le centre ancien de Barcelone, en nous basant essentiellement sur les données disponibles pour 1991, 1996, 2001 et $2005^{6}$. L'analyse a été réalisée à l'échelle des 37 zonas de recerca petita (ou ZRP), dont le découpage statistique suit plus ou moins celui des îlots urbains. Elles offrent un bon compromis entre le découpage plus fin des seccions estadístiques ${ }^{7}$, mais pour lesquelles les données statistiques sont moins riches, et celui plus lâche des quatre zones estadístiques grandes (ou ZEG) ${ }^{8}$, dont l'échelle trop petite masque des disparités très fortes entre les différents quartiers du centre ancien. Les principales hypothèses de travail ont porté sur l'amélioration des conditions de vie qui passe par une gentrification progressive du centre ancien, l'importance de l'immigration étrangère contemporaine dans la revitalisation économique et le maintien de secteurs marginalisés.

\section{LA MULTIPLICITÉ DES TRAJECTOIRES SOCIO-DÉMOGRAPHIQUES DES QUARTIERS ANCIENS DE BARCELONE}

L'interprétation des résultats des analyses statistiques plurivariées a permis de mettre en évidence la multiplicité des processus à l'œuvre entre 1991 et 2005, selon les quartiers, et la coexistence de formes de réappropriation parfois exclusives, susceptibles de générer des tensions. Les différents processus à l'œuvre sont les suivants (fig. 1).

L'analyse montre que le processus de gentrification est loin d'être généralisé dans le centre ancien de Barcelone qui comptait, en 2005, un peu plus de 110000 habitants. Il ne concerne que 7 ZRP sur 37. La reprise démographique s'accompagne d'une plus grande hétérogénéité sociale dans un contexte de croissance générale de la population et de maintien relatif des catégories socio-professionnelles dites « populaires ».

On constate au contraire qu'une grande partie du centre ancien reste caractérisée par un profil socio-démographique relativement stable entre 1991 et 2005. Il peut s'agir de certains quartiers restés populaires, surtout dans le Raval, où la part des classes populaires (ouvriers, employés) peut atteindre $70 \%$ des actifs. La proportion de non diplômés, c'est-à-dire n'ayant pas le baccalauréat ou équivalent, y est forte (parfois plus de la moitié des 16 ans ou plus) ainsi que celle de chômeurs (15,8\% en 2001). Ceci n'empêche pas d'autres formes de recomposition du quartier, avec notamment l'explosion de l'immigration étrangère depuis les années 1990. Dans certains secteurs centraux du Raval, la part des étrangers est ainsi passée de près de $6 \%$ en 1991 à quasiment $50 \%$ en 2005. Inversement, le profil socio-démographique stable peut concerner des quartiers restés aisés, par exemple dans le nord du Barrio Gótico, où la part des classes supérieures s'est maintenue autour de $30 \%$ des actifs de 1991 à 2001.

Ce qui est plus original, c'est la mise en évidence de plusieurs formes d'évolution dites de «trajectoire descendante ». Dans un premier cas, il peut s'agir de « désembourgeoisement » (trois secteurs), si l'on entend par là le remplacement de classes supérieures par des classes moyennes ou populaires. Certains entretiens nous ont confirmé que depuis quelques années les anciens

6. Pour une description précise de la méthodologie adoptée, nous renvoyons au chapitre 8 de notre thèse (Ter Minassian, 2009b).

7. «Sections statistiques». On en comptait 1919 en 1984, mais leur nombre est passé à 1917 en 2001 suite à un redécoupage dans le district de Ciutat Vella, ce qui fait qu’actuellement le centre ancien est découpé en 151 sections statistiques.

8. " Grande zone statistique ». 


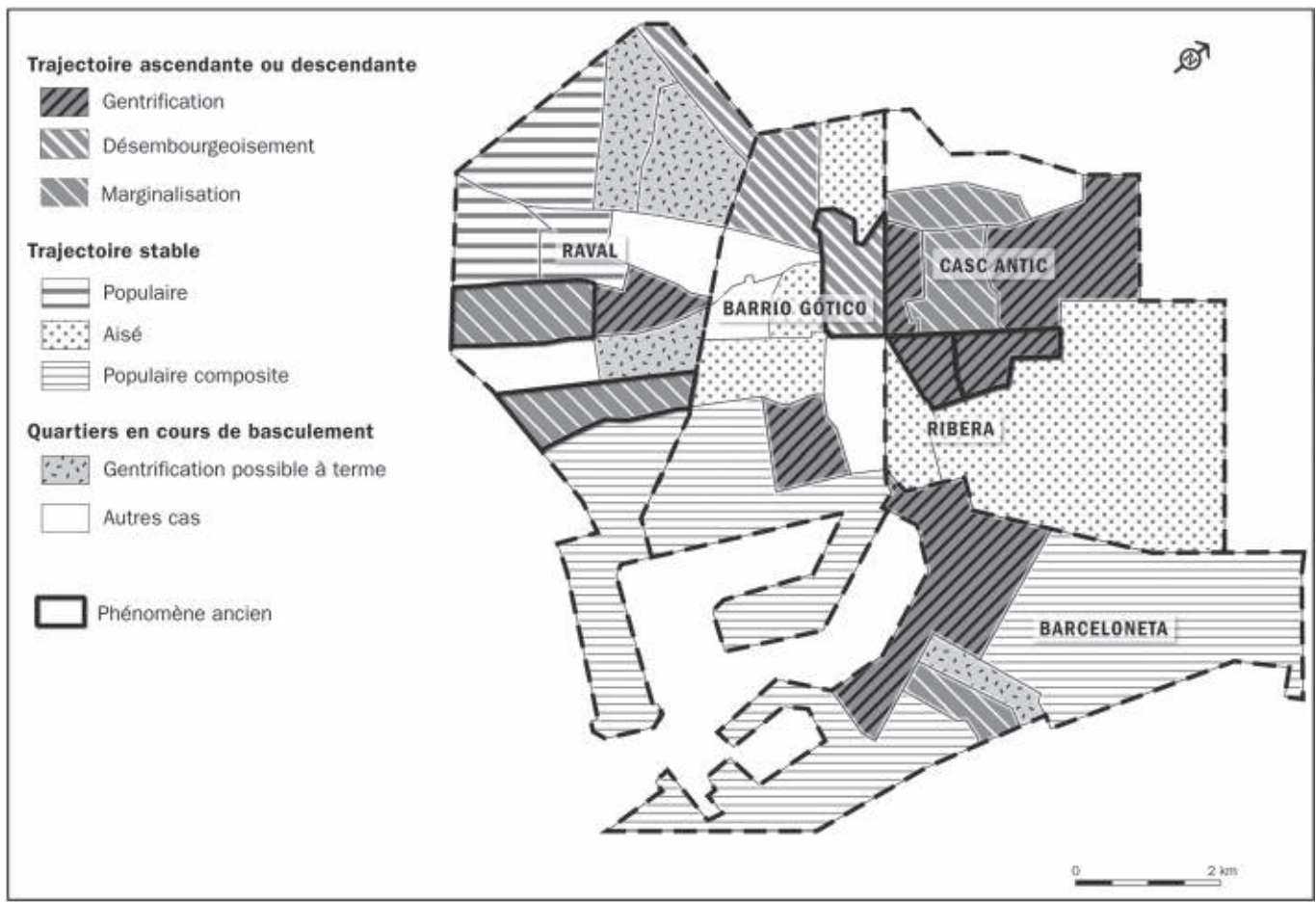

Figure 1 : Ciutat Vella entre 1991 et 2005 : un centre ancien fragmenté par de multiples processus Ciutat Vella from 1991 to 2005: an old city-centre fragmentated by multiple processes

résidents aisés, plutôt de nationalité espagnole, quittent parfois le Barrio Gótico, notamment du fait de la transformation du paysage social et économique du quartier : développement touristique jugé excessif, disparition des commerces traditionnels, immigration étrangère importante... Le second cas, de «marginalisation » (cinq zonas de recerca petita pour 17,8\% de la population), concerne des secteurs où non seulement le caractère populaire tend à se maintenir, mais où la crise socio-démographique semble même s'accentuer. Les classes populaires sont restées majoritaires (près de $69 \%$ en 2001 dans le secteur « en marginalisation » dans le sud du Raval, pour une population totale d'environ 5000 habitants) avec un taux de chômage qui reste élevé (autour de $18 \%$ en 2001) et une proportion croissante de non diplômés (d'un peu plus de $52 \%$ en 2001 à plus de $60 \%$ en 2005).

Enfin, nous avons identifié des secteurs en cours de basculement. On y perçoit bien un changement, mais l'information disponible à partir des données les plus récentes utilisées ne permet pas de trancher en faveur d'une tendance plutôt qu'une autre (quatre secteurs, pour 7,4\% de la population). Dans certains cas, un tissu social particulièrement hétérogène se maintient, mais l'apparition et la diffusion de nouveaux types de commerces, en particulier ceux destinés aux populations jeunes et/ou étudiantes, comme les bars à thème, les magasins de musique ou de vêtements, profitant notamment de la réimplantation de la faculté de géographie, d'histoire et de philosophie dans le quartier, nous incitent à parler plutôt de "gentrification de consommation ", et non de " gentrification résidentielle ${ }^{9}$ ». Une seconde hypothèse serait que la gentrification est en cours, mais plus lentement, ou bien que la tendance décelée en 2001 s'est inversée 
du fait de l'évolution socio-démographique plus récente, auquel cas nous pourrions parler de « dégentrification ». Une troisième hypothèse (non contradictoire) serait que ces quartiers suivent une trajectoire relativement similaire à ce que M. van Criekingen et J.-M. Decroly (2003) ont appelé incumbent upgrading pour décrire, à Bruxelles ou Montréal, des espaces qui connaissent peu de changements dans la composition socio-démographique de la population mais tout de même une amélioration du parc de logements du fait d'un effort de réhabilitation par les propriétaires-occupants.

\section{DES RÉAPPROPRIATIONS MULTIPLES DU CENTRE ANCIEN}

Tous ces processus à l'œuvre suscitent des formes de réappropriations qui touchent à différents aspects du cadre urbain. La première forme de réappropriation concerne le parc de logements. Nous entendons par là le fait qu'il est progressivement investi par de nouvelles populations, qu'il s'agisse des « gentrifieurs » qui récupèrent des logements vacants ou réhabilitent d'anciens logements dégradés, ou des populations jeunes et des populations immigrées, disposant souvent toutes deux de peu de moyens, qui s'orientent sur les logements les moins onéreux du centre ancien. Le parc de logements de Ciutat Vella se transforme, avec des implications sur le paysage urbain. La seconde forme de réappropriation est économique et commerciale, avec deux tendances majeures : d'une part le développement du commerce et des services touristiques, en particulier dans les quartiers les plus prestigieux du centre ancien, d'autre part l'essor du « commerce ethnique ${ }^{10}$ », dans les quartiers plus populaires ou d'immigration. La réappropriation passe aussi par le développement de nouvelles pratiques ou de nouveaux usages des quartiers anciens, du fait de l'installation de nouvelles catégories de résidents, ou bien du développement de certaines activités liées au tourisme et aux loisirs. Enfin, la dernière forme de réappropriation est symbolique, puisqu'avec l'arrivée de nouvelles populations ou le développement de nouvelles pratiques, c'est la perception du centre ancien et sa place dans l'agglomération barcelonaise qui se modifie.

La gentrification peut être considérée comme une réappropriation «totale », au sens où le processus implique toutes les formes que nous venons de décrire, avec la réhabilitation d'une partie du parc de logements, le développement de nouveaux commerces adaptés à la demande des gentrifieurs, rendant les quartiers plus « branchés » et modifiant considérablement l'image que les Barcelonais ou les touristes peuvent avoir de quartiers longtemps considérés comme mal famés. Mais on a vu que la gentrification n'était pas un processus exclusif et systématique à Ciutat Vella. L'immigration étrangère contemporaine peut être considérée comme un autre type de réappropriation «totale ", puisque ce phénomène a un impact à la fois sur le parc de logement (dont le segment le plus dégradé est récupéré par les immigrants), sur l'activité commerciale (avec le développement d'activités dédiées, comme les centres d'appel et les taxiphones), sur les pratiques dans l'espace public, et enfin sur l'image du centre ancien.

L'analyse a donc permis de mettre en évidence la multiplicité des processus dans le centre ancien de Barcelone, originaux ou bien classiques ailleurs mais prenant des formes spécifiques à Barcelone, parmi lesquels la gentrification ne constitue qu'un des phénomènes en présence ${ }^{11}$. Les processus de revalorisation ou de dévalorisation sont divers et offrent une vision beaucoup moins uniforme du cœur de l'agglomération catalane que ne le donne à voir la lecture des publications officielles. Cette coexistence de réappropriations débouche parfois sur l'émergence de phénomènes parfois inconciliables à terme, pouvant générer des tensions entre les différents groupes de population ou bien entre les populations et les pouvoirs publics, dues à la contradiction entre les attentes des résidents et la politique menée par la Municipalité. Pour certains représentants

10. On peut définir le commerce « ethnique » comme un commerce tenu par un commerçant qui n’a pas la nationalité espagnole (Serra del Pozo, 2006).

11. D'ailleurs, analysant les centres-villes de Bruxelles et de Montréal, M. van Criekingen et J.-M. Decroly (2003) arrivent à une conclusion relativement similaire (2003). 
d'associations locales de riverains ${ }^{12}$, la politique de la Municipalité n'a eu d'autre objectif que de faire de Ciutat Vella un quartier touristique, où les « résidents anciens » ont de moins en moins leur place. Pour d'autres au contraire, plutôt du côté des associations de commerçants, l'intervention publique devenait urgente pour résorber la dégradation des quartiers, en particulier dans le Raval. Mais l'opposition entre ces deux arguments n'est pas si évidente, du fait de l'ambivalence des discours de certaines associations. En réalité, les associations de riverains ont remis en cause moins l'objectif de requalification des quartiers anciens, que la méthode et les moyens mis en œuvre. Ces derniers ont, selon eux, visé à disqualifier la présence des anciens résidents. Face à ces valorisations et dévalorisations multiples du centre ancien de Barcelone, trois types de tensions ont pu ainsi émerger :

- tensions entre résidents espagnols et résidents de nationalité étrangère;

- tensions entre anciens et nouveaux résidents;

- tensions entre résidents et pouvoirs publics.

Sans chercher à être exhaustif, nous avons particulièrement mis l'accent dans le cadre de cet article sur ce dernier type de tension, qui permet d'étudier le rôle des politiques publiques dans les processus de réappropriation des quartiers anciens et d'examiner de manière critique le Modelo Barcelona.

\section{Le rôle des politiques urbanistiques dans les évolutions socio-démographiques récentes}

Pour comprendre les incidences des politiques publiques d'aménagement et d'urbanisme menées par la Municipalité sur les formes de la récupération du centre ancien de Barcelone, nous nous sommes appuyé sur l'analyse de deux projets urbains et sur des entretiens menés auprès de différentes instances représentatives de la vie locale, des associations de riverains, ainsi que quelques associations de commerçants. Ce matériau de travail a permis de mieux saisir les tensions entre résidents et pouvoirs publics, et viennent en contrepoint de la surabondance de textes publiés ou édités par la municipalité de Barcelone sur le district de Ciutat Vella.

Dans les deux secteurs d'étude, Rambla del Raval/Illa del Raval et Pou de la Figuera, l'examen met en évidence les conflits d'usages et les oppositions entre les attentes des résidents " traditionnels » et les projets municipaux. Ils se doublent dans le second cas de l'échec du processus participatif.

\section{Rambla del Raval/Illa del Raval : LA NORMalisation du tissu SOCIAL ET URbain}

Les mutations identitaires de ce secteur du Raval, quartier populaire traditionnel du centre ancien de Barcelone, révèlent les tensions sociales et symboliques générées par des réappropriations multiples, qui s'expriment à travers la transformation du paysage urbain. Dans un contexte de faiblesse de la construction de logements, la volonté de changer l'identité traditionnelle du Raval et d'en développer la fonction touristique est une orientation forte de la politique d'aménagement de la Municipalité, qui alimente une revalorisation de ces quartiers anciens, contradictoire avec les attentes des résidents « traditionnels ».

La Rambla del Raval se présente comme une grande voie occupée par un terre-plein central. Elle est un élément essentiel de la politique municipale de réhabilitation, d'une part par la superficie concernée (58 m de large pour 317 m de long), d’autre part par sa localisation au cœur du Raval. Elle a été achevée en 2000. Au total, 62 bâtiments ont été démolis pour laisser place à la Rambla, représentant 789 logements. Des 38 bâtiments situés de part et d'autre de la Rambla del Raval

12. En particulier les associations de riverains situées dans les quartiers les plus populaires du Raval et du Casc Antic. À l'occasion des entretiens que nous avons pu réaliser auprès d'elles, nous avons pu constater qu'elles rassemblaient des résidents plutôt âgés et à la retraite, le plus souvent présents dans le quartier depuis plusieurs décennies. 
en 2001, 30 ont été réhabilités intégralement ou bien étaient en cours de réhabilitation en 2001 (Focivesa, 2001), pour un total de 454 logements (Sargatal Bataller, 2003). En comparaison, on compte seulement 143 nouveaux logements sociaux, situés soit directement en bordure de la Rambla del Raval, soit à proximité (par exemple au carrefour des rues Riera Alta, Carme et Maria Aurèlia Capmany), ce qui reste largement inférieur au nombre de logements qui ont disparu dans l'opération de rénovation. Actuellement, la rénovation du secteur est complétée par celle de lîlot compris entre les rues Robador, Sant Rafael, Sant Josep Oriol et la Rambla elle-même, plus communément appelé « Illa Rambla del Raval ${ }^{13}$ » ou bien « Illa Robador ». Le projet, en voie d'achèvement, a entraîné la démolition d'anciens bâtiments dégradés de logements pour construire un hôtel de luxe, des immeubles de bureaux et de logements, et la nouvelle Cinémathèque de Catalogne.

Ici, l’objectif de la Municipalité de donner une image plus « policée » du Raval, longtemps (et encore) considéré comme un quartier populaire, de mauvaise réputation et mal fréquenté passe par une transformation de l'image du quartier, elle-même tributaire d'une redéfinition du paysage urbain. La disparition, lors de la rénovation du secteur, de la fontaine située à l'emplacement de l'actuelle Rambla del Raval, marquerait, pour une représentante d'une association de riverains du Raval, l'effacement progressif du patrimoine et de l'identité traditionnelle du quartier, et la dissolution des relations de sociabilité qui existaient dans un secteur particulièrement dense du centre ancien. Malgré l'absence de classement au titre de monument historique, on pouvait considérer cette fontaine comme un élément patrimonial, dans la mesure où elle constituait une trace de l'identité collective construite à l'échelle d'un quartier. Et on peut douter que la nouvelle fontaine, qui remplace l'ancienne déplacée dans un autre quartier de Barcelone, très impersonnelle et qui n'est absolument pas mise en valeur, puisse contribuer à la construction identitaire collective et symbolique des résidents, anciens comme nouveaux, du quartier du Raval. Le choix fait par la Municipalité de déplacer la fontaine est difficilement compréhensible, alors qu'elle affiche sa volonté de respecter l'histoire du quartier et d'être à l'écoute des anciens résidents, d'autant plus que rien n'empêchait vraiment de réinstaller la fontaine à son emplacement d'origine, une fois les travaux de la Rambla del Raval achevés. Il est donc plus plausible que ce choix s'inscrive dans une stratégie plus large de normalisation des pratiques de l'espace public au cour d'un des secteurs les plus populaires du centre ancien de Barcelone, caractéristique de l'ensemble du projet de la Rambla del Raval et de l'Illa del Raval.

L'effacement de l'identité traditionnelle du quartier du Raval s'accompagne d'un renforcement de l'activité touristique, exacerbé par la construction d'un hôtel de luxe (4 étoiles), cylindre de verre de dix étages construit sur les plans de l'architecte Pere Puig i Rodriguez, accueillant 180 chambres et 5 suites et qui a ouvert ses portes à l'automne 2008 (photo 1).

Nous pouvons d'emblée nous interroger sur la pertinence du projet global, au regard du tissu social dans lequel il s'inscrit. Dès l'origine, le projet d'hôtel a fait débat. Il est évident que sa construction ne répond pas du tout aux attentes des résidents du quartier, qu'ils soient " nouveaux » ou « anciens ». Ce sont bien les touristes qui en seront les principaux bénéficiaires (la société gérante vise en particulier le tourisme de grand luxe ou d'affaires). Sa construction peut donc être vue comme une étape supplémentaire dans le repositionnement du Raval comme une zone touristique et de loisirs à l'échelle de l'agglomération, au détriment de sa fonction résidentielle et du maintien d'une vie sociale de quartier. Par ailleurs, le style de construction choisi répond à une logique de normalisation de l'espace public, cohérente avec l'aménagement de la Rambla del Raval elle-même : la transformation d'un paysage urbain à partir d'œuvres d'art ou de bâtiments construits dans un style architectural radicalement différent de celui du quartier vise ici à modifier profondément la perception que l'on peut avoir du Raval ${ }^{14}$.

13. Illa signifie îlot en catalan.

14. Au début du projet, une vive polémique avait éclaté sur la hauteur prévue de l'hôtel. Devant les critiques des riverains mais aussi de certains professionnels de l'urbanisme, elle a finalement été revue à la baisse, de 14 à 10 étages. 


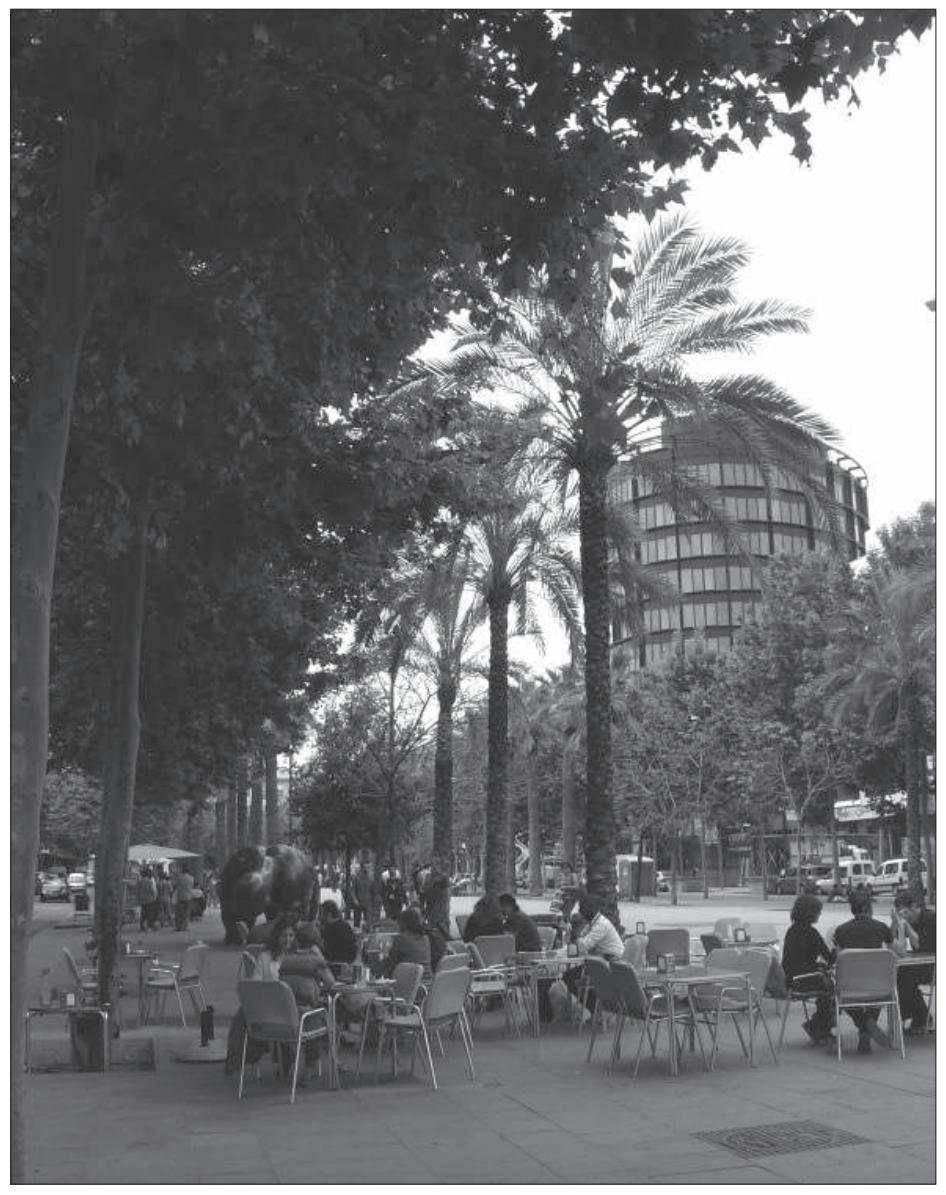

Photo 1 : Un hôtel de luxe sur la Rambla del Raval : une aberration ou un symbole du changement?

A luxury hotel on the Rambla del Raval : an aberration or a symbol of change?

Parallèlement, l'évolution récente du paysage commercial le long de la Rambla del Raval témoigne des contradictions entre l'implantation d'étrangers non européens et une amorce de gentrification commerciale. À partir d’un relevé des commerces et services situés le long de la Rambla del Raval ${ }^{15}$, nous avons pu constater le grand nombre de locaux fermés (20,3\%), ce qui témoigne des limites de la réactivation économique du quartier. La répartition spatiale des commerces et services est également digne d'intérêt. La tendance à la concentration, dans la partie du sud de la Rambla del Raval, des boucheries « halal », des restaurants rapides et d'une manière générale des commerces ethniques, s'oppose à la multiplication, dans la partie nord, des restaurants plus touristiques, des banques et des hôtels. Si la Rambla de Raval apparaît à première vue comme un espace public à forte mixité sociale, sa réalisation semble en réalité répondre beaucoup plus à une stratégie de normalisation des pratiques spatiales par diffusion de l'offre à destination des touristes et des populations « branchées ».

15. Ce travail de terrain a été réalisé en mai 2008. La date est importante : le commerce évoluant rapidement dans le centre ancien, il est possible que ce relevé soit rapidement obsolète. Néanmoins, il donne un «instantané » du commerce de la Rambla del Raval plusieurs années après la rénovation du secteur. 


\section{Pou de LA Figuera : L'ÉCHEC du PROCESSUS PARTICIPATIF}

Les enjeux de la rénovation du secteur Pou de la Figuera se posent en apparence en des termes très différents. Pour un objectif identique, celui de l'assainissement du tissu urbain, l'orientation touristique est moins évidente et le souci de répondre aux besoins des populations résidentes plus qu'à ceux des futurs hommes d'affaires qui séjourneront à Barcelone, semble plus fort. Il s'agit alors de replacer le projet dans le contexte qui l'a entouré. Les modalités de la formulation, la réalisation et la contestation du projet baptisé «Pou de la Figuera » sont révélatrices des tensions suscitées par la récupération du centre ancien de Barcelone. Elles témoignent des oppositions que peuvent faire naître les projets proposés par une Municipalité parfois insuffisamment à l'écoute des résidents. Le projet pour le secteur Pou de la Figuera résume bien toutes les contradictions d'une politique municipale, qui, malgré les déclarations d'intention, reste peu ouverte aux processus participatifs.

Entre les expropriations, la fermeture du marché pendant sept années, la perte de dynamisme commercial et le «trou de la honte », la politique de rénovation de ce secteur a été vécue par les habitants du quartier comme un traumatisme. Elle a contribué à structurer la contestation locale qui est rapidement passée de la « revendication sociale » (par exemple réclamer la construction de logements sociaux) à une revendication plus large, y compris sur le plan politique. Le projet municipal Pou de la Figuera voulait s’inscrire dans la continuité de la rénovation du marché Santa Caterina $^{16}$ et de ses abords, notamment la prolongation de l'avenue Francesc Cambó. L'opération a concerné un vaste espace de plus de $6000 \mathrm{~m}^{2}$, pour un coût total de presque 1,9 million d'euros. Elle a suscité une vive émotion et la constitution du collectif Forat de la Vergonya (« trou de la honte ») pour dénoncer le projet initial de la Municipalité qui prévoyait la création d'un espace public minéral et la construction d'un parking et d'un complexe sportif en souterrain.

Le collectif a rassemblé des résidents, à travers l'association de riverains du Casc Antic, des non résidents solidaires (à travers la fédération de l'association de riverains de Barcelone, ou FAVB), mais aussi des universitaires (Arquitectos sin fronteras) ou des associations pas forcément rattachées au territoire concerné par le projet mais généralement mobilisés sur des thématiques qui le concernent (Taller contra la violència immobiliària i urbanística, Coordinadora contra l'especulació del Raval, Recursos d'animació intercultural, cette dernière travaillant plutôt en direction des populations jeunes et des immigrés). Tous ont très vite dénoncé le projet de départ de la Municipalité, en réclamant l'abandon du parking et la construction d'un plus grand nombre de logements sociaux dans le secteur. Entre 2002 et 2004, le site Pou de la Figuera est devenu l'objet d'une véritable tentative de maîtrise du territoire par les deux parties en présence, avec d'un côté le collectif, sur le mode de l'appropriation spatiale et festive, par exemple avec la plantation d'un jardin auto-géré, et de l'autre la Municipalité, à grands renforts de barrières et de forces policières, pour empêcher l'accès au site. Parallèlement, à partir de l'été 2002, le collectif a commencé à élaborer un contreprojet face à celui de la Municipalité.

En 2005, les négociations se sont accélérées, témoignant de la part de la Municipalité socialiste d'une volonté de sortir de la crise, au moment où les médias nationaux commençaient à reprendre à leur compte l'expression «forat de la vergonya ${ }^{17}$ ». Au printemps 2005, la Municipalité a engagé le processus de participation citoyenne, avec une campagne de collecte d'opinions dans le quartier, du 13 octobre au 29 novembre 2005, dont le dépouillement a fait l'objet d'une présentation publique au début de l'année $2006^{18}$. Finalement, un nouveau projet a été rédigé et proposé, puis approuvé en conseil de district le 28 février 2006. Le projet définitif comprend,

16. Le nouveau marché a été inauguré en 2005, sur les plans de l’architecte Enric Miralles, après sept années de travaux retardés par la découverte de vestiges archéologiques et par des négociations entre la Municipalité et la Généralité en vue de l'implantation de logements dédiés aux personnes âgées.

17. Nous faisons l'hypothèse que la diffusion de l'expression forgée par le collectif auprès des médias nationaux témoigne de la réussite des différentes stratégies d'appropriation (physique et symbolique) du site Pou de la Figuera.

18. Les collectifs impliqués dans le débat se sont par la suite demandés avec perplexité pourquoi la Municipalité n’avait pas engagé le processus dès la fin des années 1990. 


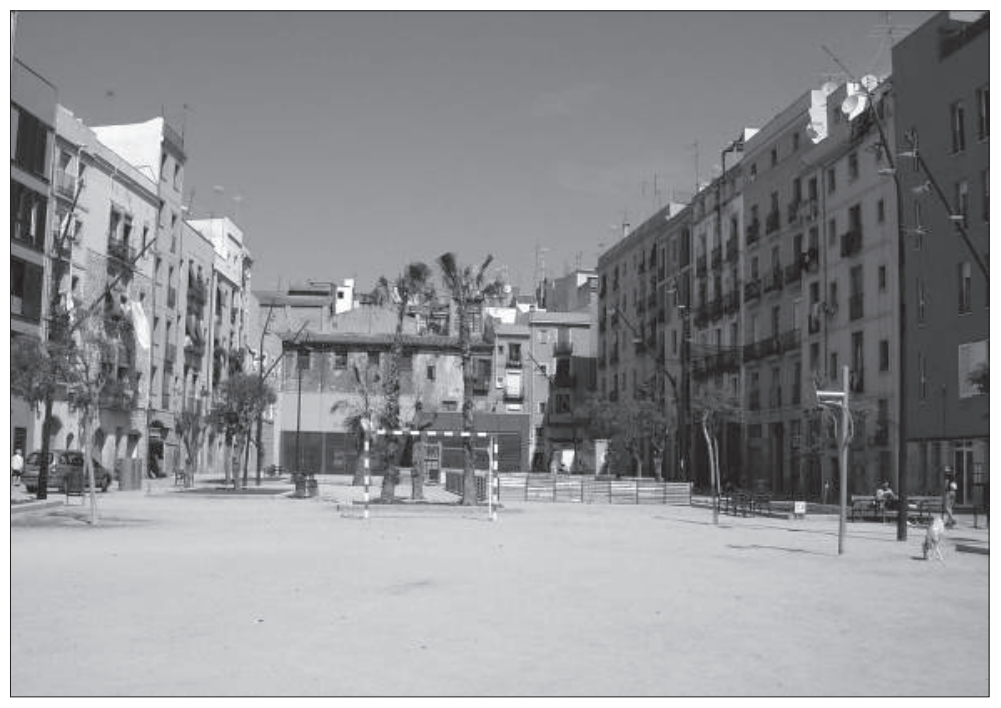

Photo 2 : La rénovation du secteur Pou de la Figuera: une récupération politique dégradée du projet des riverains

The renovation of the Pou de la Figuera sector. A degraded political recuperation of the project of the residents.

outre la modernisation de la voirie et des réseaux d'eau, de gaz et d'électricité, un nouvel espace public bordé d'arbres, un terrain polyvalent, une aire de jeux pour enfants, ainsi que deux petits bâtiments à usage collectif aux deux extrémités du site, et un immeuble de logements sociaux pour jeunes adultes, revendication portée à l'origine par le collectif Forat de la vergonya pour lutter contre la prolifération des «marchands de sommeil » dans le quartier ${ }^{19}$. Pour les pouvoirs publics, l’objectif était de créer « el nucli central de la vida de barri ${ }^{20}$ » et un «poumon vert » au cœur du centre ancien de Barcelone (Focivesa, 2006), même si la problématique d'espaces verts n’a été intégrée que tardivement et sous la pression des associations de riverains, selon une stratégie de récupération a posteriori des revendications locales (photo 2).

Il est possible de lire l'évolution des différents projets qui se sont succédé sur le site Pou de la Figuera comme des tentatives de marquage du territoire, de la part des pouvoirs publics comme des associations et des riverains qui s'opposaient au projet municipal. Chaque "marqueur ${ }^{21}$ » territorial est l'occasion de réaffirmer, pour chacun des camps en présence, sa capacité à donner du sens à l'espace urbain et à un projet socio-urbain. On peut donc questionner de deux manières différentes ces marqueurs : leur origine et leur durabilité.

Par exemple, le jardin auto-géré créé par le collectif Forat de la Vergonya est un marqueur territorial non durable. Une première tentative de jardin auto-géré a été faite en 2002, mais dispersée par la police sur ordre de la Municipalité. Après que les membres du collectif Forat de la vergonya ont finalement obtenu l'accès au site, un second jardin a vu le jour en 2004, et s'est maintenu jusqu'à l'exécution du projet définitif. Parallèlement, des manifestations, comme celle appelée « para una Ciutat Vella verde » en novembre 2002, ont été organisées pour réclamer la création d'un jardin dans un quartier qui en manquait cruellement. Mais même s'il a sans doute eu vocation à rester indéfiniment, si ce n'est au moins jusqu’à ce que la Municipalité change son projet initial et accepte l'idée de créer un jardin public, il s'est confronté à la volonté des pouvoirs publiques de se réapproprier l'espace par deux autres marqueurs non durables : d'une part les barrières policières pour empêcher l'accès au site; d'autre part, le chantier lui-même, marqueur

19. Cet immeuble comprend 24 logements sociaux en location. Sa construction a été achevée en 2006.

20. «Le noyau central de la vie du quartier».

21. Nous reprenons ici la définition de la marque proposée par V. Veschambre (2008), comme signe de la revendication de présence d'un acteur (individuel ou collectif) même en l'absence de ce dernier. 
non durable puisqu'il n'a pas vocation à rester une fois le secteur aménagé, mais qui débouche sur un autre marqueur, durable celui-là, celui du projet lui-même une fois achevé. Or, deux projets ne peuvent cohabiter sur un même site, et le projet définitif est forcément un compromis entre les deux propositions, même s'il reste à faire le bilan de ce que les pouvoirs publics ont concédé et ce que le collectif et les riverains ont réussi à faire accepter. Chez les seconds, le jardin public s'est progressivement imposé comme le principal thème mobilisateur des revendications. Au fur et à mesure que le mouvement a pris de l'ampleur, le discours s'est structuré et s'est déplacé, de la mobilisation contre les expropriations et la spéculation immobilière, à la lutte pour l'obtention d'un jardin public et d'équipements collectifs.

Certes, le projet de parking et de salle de sports en souterrain a été assez rapidement abandonné et son retrait ne constitue pas une réelle «victoire », tant la Municipalité semblait elle-même n'y accorder qu'un intérêt relativement limité. Elle a donc rapidement cédé sur des propositions qu'elle ne comptait de toute façon pas maintenir. Mais si le projet définitif reprend dans ses grandes lignes l'utilisation de l'espace qui a caractérisé la période « auto-gérée » du site, certains ajustements traduisent la volonté des pouvoirs publics de rester maîtresse du projet. Il faut peutêtre voir dans le fait que la Municipalité a choisi de substituer de nouvelles essences d'arbres à la place de celles plantées par les résidents eux-mêmes, une manière de normaliser l'espace et de réaffirmer, de manière symbolique et malgré tous les compromis qu'elle a pu faire, qu'en matière d'aménagement, c'est elle qui a le dernier mot. De même, la disposition des équipements, dans le projet définitivement adopté, diffère des propositions émises par le mouvement. Le terrain de basket, initialement situé dans la partie sud, disparaît pour laisser place à un petit espace arboré ; le terrain de football est remplacé par une piste polyvalente. De plus, la Municipalité n'a conservé pratiquement aucune des installations construites par le collectif (en matériaux de récupération). Du projet de jardin auto-géré il reste encore la huerta communautaria ${ }^{22}$, dont le comité de gestion se réunit tous les quinze jours dans le local situé au nord du secteur.

\section{Conclusion}

L'analyse que nous avons menée ne permet pas d'expliciter clairement le lien entre politiques publiques et processus de dévalorisation. En ce qui concerne la marginalisation, on peut supposer que l'effet d'entraînement escompté par la concentration spatiale et temporelle des projets d'aménagement ou de rénovation urbaine n'a pas eu lieu. Quant au désembourgeoisement de certains secteurs plus prestigieux du Barrio Gótico, on peut également penser que c'est l'ensemble de la politique publique à l'égard du district de Ciutat Vella qui a joué ici, suscitant un développement excessif de l'activité touristique qui semble, parmi d'autres facteurs, motiver le départ de certains résidents aisés de Ciutat Vella vers d'autres quartiers de Barcelone.

En revanche, l'étude de deux secteurs d'opérations d'aménagement du centre ancien de Barcelone (Rambla/Illa del Raval et Pou de la Figuera) a montré que parmi l'ensemble des processus socio-démographiques qui affectent le district de Ciutat Vella depuis les années 1990 et que nous avons présentés dans la seconde partie de notre texte, certains sont bien alimentés par les politiques d'aménagement et d'urbanisme menées par la Municipalité. La vocation touristique attribuée au Raval, associée à une volonté d'en changer l'identité pour en donner une image moins sulfureuse, favorise une gentrification de ces quartiers, qui reste cependant encore limitée. Plusieurs hypothèses peuvent l'expliquer, notamment la forte présence de l'immigration étrangère, qui alimente la concurrence sur les logements bon marché, et le maintien d'une mauvaise image qui semble encore détourner une partie des classes moyennes du parc de logements du centre ancien (Ter Minassian, 2009a). Dans le cas du projet dans le secteur Pou de la Figuera, l'échec du processus participatif témoigne du fait que la Municipalité de Barcelone semble plus soucieuse de soigner son image que de répondre aux attentes des résidents. 
Le lien observé des rapports entre l'action des pouvoirs publics et les changements sociaux dans le centre ancien de Barcelone autorise une lecture politique de l'évolution socio-démographique de Ciutat Vella. Ou bien l'on considère que la politique menée par les pouvoirs publics a bien été une politique de gentrification des quartiers anciens, mais que celle-ci a en partie échoué - du fait de la persistance d'une mauvaise image du centre et de la mobilisation des riverains contre des projets jugés inadaptés aux besoins des résidents « traditionnels » et avant tout destinés aux « nouveaux » résidents - ou bien l'on considère au contraire que l'absence de gentrification est le signe de la réussite de la politique menée. Si l'on peut constater qu'une grande partie de Ciutat Vella (sans doute à l'exception du Barrio Gótico) ne connaît pas de «muséification » ou de gentrification, l'hypothèse d'une «troisième voie » dans les politiques de réhabilitation des centres anciens, qui correspondrait au Modelo Barcelona, paraît difficile à valider. Ses spécificités tiennent peut-être plus à un décalage des phénomènes dans le temps, ou à des facteurs limitants propres à l'agglomération catalane, qu'à une politique urbanistique réellement originale. Dans cette situation, on peut se demander comment la Municipalité entend garantir l'accueil des étrangers non européens dans le centre ancien, faciliter le maintien des « résidents traditionnels » menacés par les expropriations et l'élévation rapide des loyers, et enfin éviter un développement excessif des fonctions touristiques et commerciales, au détriment de la fonction résidentielle.

\section{Bibliographie}

Ajuntament de Barcelona, 1970. Revisión del Plan Comarcal de 1953, Barcelone, Ajuntament de Barcelona, $141 \mathrm{p}$.

Ajuntament de Barcelona, 1991. Primeres jornades Ciutat Vella, Barcelone, Ajuntament de Barcelona, p. 29-37.

Andreu M., 2008. Moviments socials i crítica al "modelo Barcelona". De l'esperança democràtica de 1979 al miratge olímpic de 1992 i la impostura cultural del 2004, Scripta Nova, 2008, vol. XII, n 270 (119).

Bailey N., Robertson D., 1997. Housing renewal, urban policy and gentrification, Urban Studies, vol. 34, $n^{\circ} 4$, p. 561-578.

Bidou-Zachariasen C. (dir.), 2003. Retours en ville, Paris, Descartes \& Cie, 267 p.

Borja J., 2004. Barcelona y su urbanismo. Éxitos pasados, desafíos presentes, oportunidades futuras, dans Borja J., Muxí Z. (dir.), Urbanismo en el siglo XXI. Bilbao Madrid Valencia Barcelona, Barcelone, Edicions UPC/ETSAB, p. 171-181.

Bourdin A., 1984. Le patrimoine réinventé, Paris, PUF, 239 p.

Capel H., 2005. El Modelo Barcelona. Un examen critíco, Barcelone, Ediciones del Serbal, 117 p.

Castells M., 1974. Planificación urbana y movimientos sociales : el caso de la renovación urbana en Paris, dans Béringuier C., Castells M., Mingasson C., Rémy J., Urbanismo y práctica política, Barcelone, Los Libros de la Frontera, p. 125-160.

Clenval A., 2008. La gentrification des quartiers populaires à Paris : dynamiques spatiales, identité sociale et politiques publiques, thèse de géographie sous la direction de Catherine Rhein et de Pétros Petsimeris, Université Paris 1, 602 p.

Coing H., 1966. Rénovation urbaine et changement social, Paris, Éditions Ouvrières, 296 p.

Focivesa, 2001. Illa i Rambla del Raval. 1988 un proyecto, 2001 una realidad, Barcelone, Focivesa, n.p.

Focivesa, 2006. Santa Caterina i Sant Pere, Barcelone, Focivesa, 15 p.

Garcia i Miquel E., Vilanova i Clarer J., 1991. Distribució territorial de la població i de les activitats econòmiques, dans Ajuntament de Barcelona, Primeres jornades Ciutat Vella, Barcelone, Ajuntament de Barcelona, p. 29-37.

GLass R., 1964. Introduction to London : aspects of change, Londres, Center for Urban Studies and Mac Gibbon and Kee, 343 p.

Goмà R., 1997. Degradació, crisi urbana i regeneració a Ciutat Vella de Barcelona : una anàlisi políticoespacial, Documents d'Anàlisi Geogràfica, n 30, p. 65-92. 
LÉvY J.-P., 1987. Centres villes en mutation, Paris, CNRS, 257 p.

Préteceille E., 2007. Is gentrification a useful paradigm to analyse social changes in the Paris metrópolis?, Environment and Planning A, vol. 39, $\mathrm{n}^{\circ}$ 1, p. 10-31.

Procivesa, 2003. Ciutat Vella ciutat construida, Barcelone, El Cep i la Nansa, 269 p.

Sargatal Bataller M. A., 2003. La vivienda en el centro histórico de Barcelona. El caso de la Rambla del Raval, Scripta Nova, vol. VII, n 146 (069).

SAVARY S., 2005. Imaginaires d'une ville: Barcelone par ses paysages. Une étude géolittéraire, thèse de géographie sous la direction de Yves Luginbühl, Université Paris 1, 3 vol., 1056 p.

Serra del Pozo P., 2006. El comercio étnico en el distrito de Ciutat Vella de Barcelona, Barcelone, Fundación «La Caixa », 189 p.

Ter Minassian H., 2009a. Y a-t-il une gentrification à Barcelone? Le district Ciutat Vella entre 1991 et 2005, Geocarrefour, vol. 84, n 1-2, p. 93-103.

Ter Minassian H., 2009b. Ciutat Vella entre réhabilitation et gentrification. Politiques publiques et changements sociaux dans le centre ancien de Barcelone (1980-2008), thèse de géographie sous la direction de Martine Berger et Horacio Capel, Université Paris 1, Universitat de Bacelona, 571 p.

van Criekingen M., Decroly J.-M., 2003. Revisiting the diversity of gentrification : neighbourhood renewal processes in Brussels and Montreal, Urban Studies, vol. 40, n 12, p. 2451-2468.

Veschambre V., 2008. Traces et mémoires urbaines. Enjeux sociaux de la patrimonialisation et de la démolition, Rennes, PUR, 315 p.

Von Heeren S., 2002. La remodelación de Ciutat Vella. Un análisis crítico del modelo Barcelona, 129 p.

Cet article a été reçu le 15 avril 2010 et définitivement accepté le 16 novembre 2010. 